\title{
NEXT, a HPXe TPC for neutrinoless double beta decay searches
}

\author{
Justo MARTIN-ALBO* \\ Instituto de Física Corpuscular (IFIC), CSIC - Univ. de Valencia \\ E-mail: Justo.Martin-Albo@ific.uv.es
}

The next-generation experiments for neutrinoless double beta decay searches are a major challenge, since the detectors have to fulfill conflicting requirements: they have to be very massive and scalable; very sensitive to the signature of the $\beta \beta$ processes, and simultaneously impose extremely stringent limits on the copious backgrounds.

A high-pressure gaseous Xenon TPC may be the ideal detector for this purpose: the isotope ${ }^{136} \mathrm{Xe}$ is almost $9 \%$ of natural Xenon and enrichment by centrifugation is a relatively easy technology as demonstrated by the EXO collaboration; the detector can be extrapolated to large masses; energy resolution in gas is expected to be at the level of $1 \%$ FWHM at $Q_{\beta \beta}$; this fact, combined with the very long life of the $\beta \beta^{2 v}$ mode accounts for negligible backgrounds of intrinsic origin up to masses of the order of 1 ton. The detector is fully active and the gas can be continuously re-circulated and purified, which, together with the existence of a kinematical signature gives an extra handle against backgrounds.

NEXT (Neutrino Experiment with a Xenon TPC) is an on-going project that aims to build a 100 $\mathrm{kg}$ HPXe TPC at a pressure of $\sim 10$ bar. The experiment has already been aproved by the scientific committee of the Canfranc Underground Laboratory (LSC), in Spain, for the initial R\&D phase. The NEXT Collaboration has defined an ambitious roadmap for the next five years: an initial phase of R\&D during 2 years will be used to decide the technology (MicroMegas, GEMs, APDs, PMTs, etc.); in 3 years a first prototype (NEXT-10) will be constructed; followed by NEXT-100 in 5 years. A special grant (Consolider-Ingenio 2010) from the Ministry of Science of Spain will fund the experiment during this period. The Collaboration is composed by the leading Spanish groups in experimental neutrino and underground physics.

The general ideas behind neutrinoless double beta decay searches with a HPXe TPC are discussed in this note.

10th International Workshop on Neutrino Factories, Super beams and Beta beams

June 30 - July 52008

Valencia, Spain

\footnotetext{
${ }^{*}$ On behalf of the NEXT Collaboration.
} 
Neutrinos, unlike the other Standard Model fermions, could be Majorana particles, that is, truly neutral particles identical to their antiparticles. Majorana neutrinos provide an attractive explanation for the smallness of neutrino masses, the so-called seesaw mechanism. Besides, Majorana neutrinos violate lepton-number conservation. This, together with CP-violation, is a basic ingredient to help uncover the reasons why matter dominates over antimatter in our Universe.

Neutrinoless double beta decay $\left(\beta \beta^{0 v}\right),(Z, A) \rightarrow(Z+2, A)+2 e^{-}$, is possible if and only if neutrinos are Majorana particles. The observation of such a process is so far the only experimental method to reveal the nature of the neutrino. Furthermore, the measurement of the lifetime for this process would provide direct information on the absolute scale of neutrino masses.

The half-life of the $\beta \beta^{0 v}$ process, if mediated by the exchange of a light Majorana neutrino, is inversely proportional to the square of the effective Majorana mass, $\left\langle m_{v}\right\rangle$, defined as the $m_{11}$ element of the neutrino matrix. $\left\langle m_{v}\right\rangle$ can be written in terms of the known values of the neutrino oscillation parameters to deduce a range of possible values, as illustrated in Figure 1(a).

To explore the degenerate hierarchy of neutrino masses - corresponding to an effective neutrino mass up to $50 \mathrm{meV}$ - an $\mathrm{O}(100) \mathrm{kg}$ experiment will be needed. If no signal is found, the inverse hierarchy, extending from 20 to $50 \mathrm{meV}$, will be accessible only to experiments that can simultaneously achieve large fiducial mass and effectively no backgrounds.

\section{Xenon-based experiments for $\beta \beta^{0 v}$ searches}

The search for $\beta \beta^{0 v}$ processes has been traditionally carried out with devices such as germanium calorimeters $[1,2]$, characterized by high efficiency for the signal and outstanding energy resolution. These experiments are nevertheless background-dominated since any energy deposition in the end-point region would fake the signal. Other experiments such as the NEMO series [3] were designed to exploit the topological signature of a $\beta \beta$ event as an additional handle to reject backgrounds, but their energy resolution and efficiency are modest.

We believe that a Xenon-based experiment offers the best compromise between these conflicting requirements. Xenon is a noble gas, so can be used to track particles. It does not have radioactive isotopes other than the $\beta \beta^{2 v}$ decaying ${ }^{136} \mathrm{Xe}$. It can be enriched by centrifugation methods to high concentrations of ${ }^{136} \mathrm{Xe}$. Its $Q_{\beta \beta}$ is acceptably high and the $\beta \beta^{2 v}$ mode, not yet measured, may be as long as $10^{22}-10^{23}$ years, while the $\beta \beta^{0 v}$ mode is predicted to be about as good as the one of ${ }^{76} \mathrm{Ge}$. Finally, TPCs are well-known detectors that can be extrapolated to large masses.

In the design of a Xenon-based TPC, one can consider two options: liquid-Xenon (LXe), as adopted by the EXO collaboration [4], or high-pressure gas-Xenon (HPXe), technique pioneered by the Gotthard experiment [5]. Compactness is the obvious advantage in the use of a LXe TPC. On the other hand, in a HPXe electron tracks are long enough to be identified, offering an additional handle to reject backgrounds.

\section{The NEXT concept}

The search for neutrinoless double beta decay is about suppressing backgrounds. The detector has to be very sensitive to the signature of the $\beta \beta^{0 v}$ processes, providing at the same time handles 


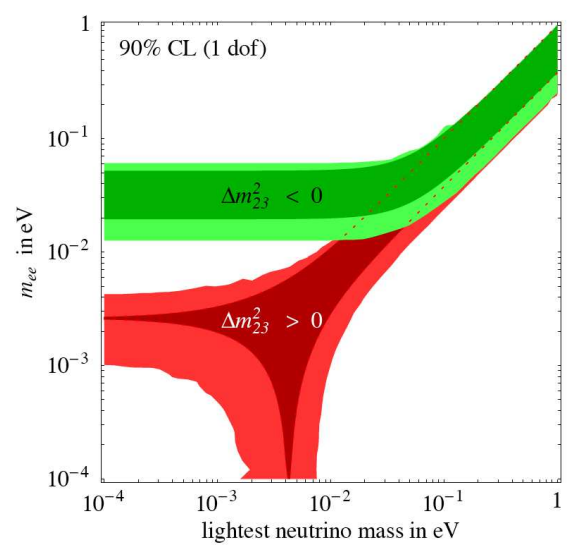

(a)

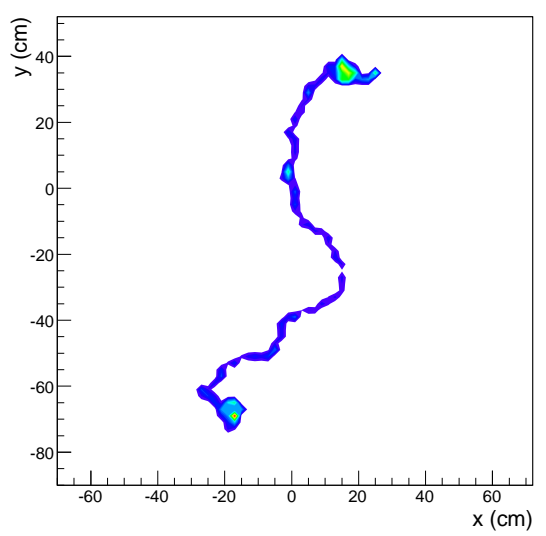

(b)

Figure 1: (a) 90\% CL range for the effective Majorana mass $\left\langle m_{v}\right\rangle$ as a function of the lightest neutrino mass. Red, for the normal hierchary. Green, for the inverted hierarchy. From [6]. (b) Topological signature of a $\beta \beta$ event: a track with energy "blobs" in both ends.

to reject the numerous backgrounds.

Good energy resolution is a must to prevent backgrounds, specially those events from the tail of the $\beta \beta^{2 v}$ spectrum falling in the window of interest. Latest developments on TPC technology may do feasible to reach energy resolutions close to the intrinsic limit in HPXe.

Detection of the initial scintillation prompt signal of an event and its use as initial trigger provides complete 3D-reconstruction of the events, which allows the rejection of essentially all charged particle backgrounds. The most serious background for NEXT are high-energy gamma rays from the natural radioactive chains, specially the ${ }^{208} \mathrm{Tl} 2.6 \mathrm{MeV}$ gammas. However, the tracking capabilities of the NEXT TPC add an additional rejection factor based on a topological criterion: $\beta \beta$ events display "blobs" at both ends of the event track (see Figure 1(b)). Therefore, most of the $\gamma$-background events can be distinguished by the presence of multiple Compton scatters, single blob topologies, K-shell fluorescence, etc.

A description of the R\&D tasks towards the NEXT detector is also given in these proceedings [7].

\section{References}

[1] H.V. Klapdor-Kleingrothaus et al., Eur. Phys. J. A. 12 (2001) 147.

[2] C.E. Aalseth et al., Phys. Rev. C 59 (1999) 2108.

[3] R. Arnold et al., Nucl. Inst. Meth. A 536 (2005) 79.

[4] EXO Collaboration, Letter of Intent, [http://www-project. slac.stanford.edu/exo/].

[5] R. Luscher et al., Phys. Lett. B 434 (1998) 407.

[6] F. Feruglio, A. Strumia and F. Vissani, Nucl. Phys. B 637 (2002) 345 [arXiv:hep- ph/0201291].

[7] M. Ball, PoS(Nufact08)119. 